\section{$\underset{\substack{\text { hommes } \\ \text { \& migrations }}}{ }$}

\section{Hommes \& migrations}

Revue française de référence sur les dynamiques

migratoires

\section{$1299 \mid 2012$}

Musulmanes et féministes en Grande-Bretagne

\title{
Le défi de la sécurité
}

Les activistes musulmanes en Grande-Bretagne

\section{Laura Zahra McDonald}

Traducteur : Françoise Bouillot

\section{(2) OpenEdition}

Journals

\section{Édition électronique}

URL : http://journals.openedition.org/hommesmigrations/1405

DOI : 10.4000/hommesmigrations. 1405

ISSN : 2262-3353

\section{Éditeur}

Musée national de l'histoire de l'immigration

Édition imprimée

Date de publication : 1 septembre 2012

Pagination : 44-53

ISSN : 1142-852X

Référence électronique

Laura Zahra McDonald, «Le défi de la sécurité », Hommes \& migrations [En ligne], 1299 | 2012, mis en

ligne le 01 septembre 2014, consulté le 03 mai 2019. URL : http://journals.openedition.org/

hommesmigrations/1405; DOI : 10.4000/hommesmigrations.1405 


\section{Le défi de la sécurité Les activistes musulmanes en Grande-Bretagne}

Par Laura Zahra McDonald, chercheure, université de Birmingham

Le climat sécuritaire qui s'est installé en Grande-Bretagne après les attentats de 2001 et $\mathbf{2 0 0 5}$ a profondément modifié le visage de la société britannique. La "guerre contre la terreur" devient le mot d'ordre de l'État britannique. Par la force de leur engagement social et politique et la qualité de leurs réseaux, les activistes musulmanes sont en première ligne de ce combat. Cette opportunité pour se faire entendre dans le débat public a néanmoins une contrepartie : le risque de l'instrumentalisation. 
En Grande-Bretagne, dans le contexte de l'après-11 septembre, le discours politique sécuritaire de guerre à la terreur présente les communautés musulmanes comme suspectes et potentiellement dangereuses. L'activisme musulman social et politique est passé au crible sous l'angle de la sécurité, évalué et jugé en fonction de l'aide ou des entraves qu'il peut apporter à la lutte de l'État contre le terrorisme. L'État britannique vise donc à promouvoir ou à exclure diverses formes d'activisme musulman, dont celles qui impliquent les femmes musulmanes, dans le cadre d'une politique de prévention antiterroriste. Il s'agit d'analyser les défis auxquels sont confrontées les femmes musulmanes du fait de ce climat sécuritaire et des politiques antiterroristes. Les militantes sont en effet à l'intersection d'une série d'interactions complexes entre l'État britannique et les citoyens musulmans, qui peuvent sembler bénéfiques pour les diverses causes qu'elles défendent, mais finissent par entraver et politiser leur travail. Ces observations sont issues d'une longue recherche qualitative financée par l'Arts and Humanities Research Council, à l'université de Birmingham, portant sur l'engagement de l'État et des musulmans dans la politique sécuritaire et l'anti-terrorisme ${ }^{(1)}$. L'auteure a développé des analyses centrées sur le genre dans le cadre de cette recherche.

\section{L'activisme des femmes musulmanes britanniques}

Le discours public britannique, embrassant un modèle de type multiculturel, reconnaît explicitement des formes d'expression culturelle collectives et individuelles multiples, et en tient compte. Dans ce cadre, la catégorie "femme musulmane britannique" correspond à une identité partagée dont le mode d'organisation sociale et politique repose sur des affiliations de genre, sur la foi en l'islam et sur la citoyenneté. Cette catégorie indique à la fois les frontières de l'auto-identification et les ascriptions de ceux qui lui sont extérieurs. C'est une catégorie très large, surtout au vu de la diversité ethnique, culturelle, sociale et religieuse de la population musulmane britannique, et plus encore du point de vue postmoderne qui reconnaît le pluralisme et l'individualité. Et pourtant, les femmes musulmanes britanniques partagent des composantes importantes de leur expérience, et le choix de s'organiser selon cette catégorie exprime en partie la tension entre action individuelle et "essentialisme stratégique $^{(2)}$. Les femmes musulmanes en Grande-Bretagne affrontent une exclusion structurelle et sociale, qui va de la discrimination au travail ${ }^{(3)}$ jusqu'à l'incitation à la haine raciale et religieuse, délit puni par la loi britannique, à cause de la perception de leur identité personnelle ${ }^{(4)}$. En réaction, les activistes qui s'identifient comme musulmanes et abordent les questions de foi et de genre affectant les femmes ont 
fondé ces dernières années des organisations au niveau local, national et international, avec une forte présence sur le Net. Leurs cibles et leurs méthodologies sont diverses, dont certaines sont spécifiques à une démographie particulière, ou se concentrent sur des objectifs particuliers, alors que d'autres visent au contraire à créer des réseaux plus vastes relayant la voix des femmes musulmanes. C'est un travail à long terme qui promeut une participation et une inclusion politiques et civiques pour lutter contre l'orientalisme et l'islamophobie dans la société britannique, et pour défier les pratiques communautaires, notamment les interprétations patriarcales de l'islam et les délits ou crimes commis au nom de la culture, comme les mariages forcés. Le financement et les affiliations de cet activisme sont très divers : les organisations indépendantes en Grande-Bretagne s'appuient souvent sur un mélange précaire de donations caritatives, de travail bénévole et de financements gouvernementaux et non gouvernementaux, en insistant sur la transparence et la traçabilité des fonds. Ce financement est lié en général aux agendas sociaux et politiques, aux intérêts ou aux objectifs des financeurs. Quant aux financements gouvernementaux, ils sont traditionnellement liés à la promotion de l' intégration", de la cohésion et du développement communautaire, de l'égalité et de la régénération locale. Cependant, les intérêts et le financement du gouvernement britannique se sont considérablement modifiés à la suite des événements du 11 septembre 2001.

\section{L'activisme à l'ère de la nouvelle terreur}

À la suite du 11 septembre, et plus encore depuis les attentats de Londres de juillet 2005, où de jeunes musulmans britanniques ont commis des attentats suicides dans leur propre pays, le gouvernement britannique a adopté des concepts politiques et idéologiques alignés sur la notion de guerre au terrorisme. Dans ce contexte, la communauté musulmane a été désignée comme l'ennemi et le danger, et la politique gouvernementale vis-à-vis des communautés musulmanes a accru son volet sécuritaire. Ce mécanisme sécuritaire tous azimuts s'est construit selon la stratégie britannique de contre-terrorisme CONTEST, qui a constitué une antenne de prévention vis-à-vis des communautés “à problèmes”. Le programme Prevent, depuis sa conception en 2005, a donc influencé une série de thèmes politiques, qui vont de l'éducation - contre la radicalisation - jusqu'à l'“empowerment", c'est-à-dire l'autonomisation des communautés dans le but de résister à la rhétorique terroriste et de susciter un flux d'informations destiné à la police et au public ${ }^{(5)}$. Cette obsession de la sécurité et la perception des communautés musulmanes comme le lieu d'un extrémisme potentiel ont fortement tendu et politisé la relation entre l'État britannique et les citoyens 
musulmans. On a pu constater une montée de la peur chez les citoyens musulmans perçus comme un groupe suspect ${ }^{(6)}$. Cette peur a été exacerbée par des expériences directes - les jeunes musulmans ont subi une recrudescence de vérifications policières depuis $2005^{(7)}$ - et par le discours public, le Premier ministre David Cameron ayant contribué directement à la stigmatisation en associant le processus complexe de radicalisation violente à une absence d'intégration sociale des musulmans ${ }^{(8)}$. Quant aux organisations musulmanes, la politique gouvernementale n'a eu de cesse de les classer de façon simpliste entre celles avec qui il est possible de travailler et celles qu'il convient d'exclure, créant ainsi l'image du "bon et du mauvais musulman”. Les groupes jugés théologiquement ou politiquement problématiques par les politiciens se retrouvent
À la suite du 11 septembre, et plus encore depuis les attentats de Londres de juillet 2005, où de jeunes musulmans britanniques ont commis des attentats suicides dans leur propre pays, le gouvernement britannique a adopté des concepts politiques et idéologiques alignés sur la notion de guerre au terrorisme. exclus et étiquetés comme extrémistes, non seulement au détriment des voix qu'ils représentent, mais du débat social et politique en général. Dans ce contexte, l'intérêt du gouvernement pour les organisations de base s'est transformé en une attention active et politisée au sein d'un cadre presque exclusivement sécuritaire.

\section{Les femmes musulmanes dans les politiques anti-terroristes}

L'intérêt pour l'activisme communautaire musulman est issu des leçons apprises en Irlande, à savoir que "les communautés sont la clé de la défaite du terrorisme". Associé à la politique de Prevent, l'antiterrorisme a été si bien institutionnalisé que diverses tactiques de prévention de la radicalisation, de déradicalisation et de vigilance vis-àvis des activités liées au terrorisme sont dirigées contre les communautés musulmanes en général, et contre des groupes cibles spécifiques. Sous les gouvernements travaillistes de Tony Blair et de Gordon Brown, la politique de Prevent a explicitement identifié les femmes musulmanes comme un groupe à coopter pour servir l'antiterrorisme, leur genre et leur foi inspirant au gouvernement certains présupposés, certaines attentes et donc des offres spécifiques. C'est là un grand changement dans la représentation classique qu'ont les Occidentaux des femmes musulmanes : les domaines du 
terrorisme et de l'antiterrorisme sont en général perçus comme masculins, associés à une perception populaire, politique et académique des femmes qui seraient des victimes passives, les femmes musulmanes étant considérées encore davantage comme des victimes par excellence du patriarcat, de la guerre et de la violence terroriste. Même lorsqu'elles sont actives dans le terrorisme, les femmes sont perçues comme déviantes, poussées à la violence pour des raisons irrationnelles, sociales ou émotionnelles, plutôt que par des objectifs politiques "masculins ${ }^{(9)}$ ". Par exemple, les "veuves noires" de Tchétchénie et les Palestiniennes ayant commis des attentats suicides sont mises à la même enseigne, malgré la différence des contextes. Leur violence est définie par le genre, il s'agit donc d'une victime féminine dont la violence résulte de l'exploitation (masculine) du deuil, du déshonneur et de la vengeance. Les références explicites quant à la pertinence des femmes musulmanes dans la politique antiterroriste britannique indiquent une différence intéressante avec les constructions issues de la perception ordinaire des femmes activistes. Dans les premières moutures de cette stratégie, les femmes étaient censées "construire une résilience dans les communautés" et "soutenir les voix modérées ${ }^{(10) "}$ - ce qui leur présuppo-

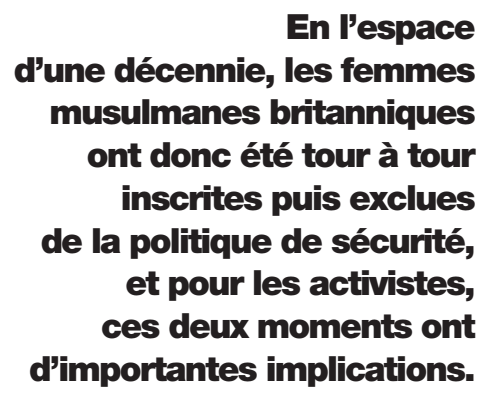

sait un sens inné de la modération et une forme de pouvoir dans leur communauté. On attendait donc d'elles qu'elles soient une passerelle vers l'État en vertu de leur genre et de leur position en tant que mères, épouses et sceurs d'hommes musulmans. Les projets centrés sur les femmes étaient financés au niveau local dans les secteurs des villes jugés les plus vulnérables à l'extrémisme violent. Ils comportaient la formation des femmes à l'usage d'Internet - au cas où des membres de la famille consulteraient des sites terroristes - et la construction de réseaux de femmes musulmanes engagées dans des activités anti-extrémistes. Pour une meilleure efficacité, le Department of Communities et le Local Government ont fondé le National Muslim Women's Advisory Group (NMWAG), un petit groupe de militantes musulmanes expérimentées qui étaient censées agir en tant que représentantes de leurs communautés. Mais, depuis 2011, le gouvernement conservateur de David Cameron a revu cette politique, jugeant que les objectifs de Prevent, y compris le financement de groupes de femmes, devaient cibler l'intégration et la cohésion, et non l'antiterrorisme ${ }^{(11)}$, l'objectif implicite étant de se concentrer sur les vues et comportements extrémistes d'individus et de groupes spécifiques, plutôt que sur des communautés entières. En l'espace d'une 
décennie, les femmes musulmanes britanniques ont donc été tour à tour inscrites puis exclues de la politique de sécurité, et pour les activistes, ces deux moments ont d'importantes implications.

\section{Soutien gouvernemental et instrumentalisation}

Pour les organisations de femmes musulmanes, le financement étatique pour des projets promouvant l'apprentissage et la participation était une épée à double tranchant. Le financement, qui pose toujours un problème pour les communautés locales, était généreux, et les conditions qui lui étaient associées - aider à protéger les communautés de l'extrémisme violent - étaient bénéfiques à la sécurité de l'État comme à celle des communautés. Après tout, qui n'est pas contre la violence et pour la protection de la vie ? En outre, les autorités gouvernementales et locales ont commencé à s'intéresser à des groupes ayant longtemps travaillé sans être vraiment reconnus : avec l'argent de Prevent, les conseils municipaux ont pu commander des recherches et faciliter les réunions et les réseaux de femmes musulmanes. De plus, les questions jusque-là traitées uniquement par les activistes se sont soudain retrouvées en tête de l'agenda des membres du cabinet - notamment l'accès aux mosquées et la participation à leur gestion, la direction de la communauté et l'éducation des adultes. Ces opportunités de rendre plus visibles certaines organisations semblaient une étape pour une meilleure représentation des femmes musulmanes de la base.

Les préoccupations sécuritaires ont aussi contraint les activistes - qu'elles soient ou non d'accord avec la politique gouvernementale - à s'engager davantage sur les questions d'interprétation théologique, sur les processus de radicalisation violente et sur des questions communautaires sous-jacentes comme l'aliénation politique et sociale de certains jeunes musulmans britanniques. Elles ont galvanisé de petits groupes pour développer des compétences et avoir un impact direct sur la prévention de l'extrémisme violent. Ces groupes, y compris certaines mosquées, avaient abordé ces questions bien avant le 11 septembre, créant des approches communautaires innovantes pour combattre le terrorisme ${ }^{(12)}$ : avec le programme Prevent, ce travail hautement sensible et nécessitant une profonde connaissance des questions sociales, théologiques et politiques, a soudain pris de la valeur et a été soutenu. Ce travail s'est penché sur les expériences d'individus vulnérables et a défié les sympathisants du terrorisme. Au sein de ces groupes, les femmes activistes ont commencé à aborder des questions liées à la prévention de l'extrémisme violent dans des contextes souvent inaccessibles à leurs homologues masculins. Elles travaillaient avec les familles d'individus condamnés, avec des détenues inculpées sous le coup des lois antiterroristes ou jugées vulnérables 
à l'extrémisme violent dans la prison, et avec les femmes de communautés ayant des sympathies pour Al Qaeda ou des groupes du même type. Les femmes ont aussi mené des campagnes et fondé des groupes de pression pour combattre l'injustice émanant de l'État, surtout à cause des lois antiterroristes, des infractions aux droits de l'homme et de la politique étrangère - éléments alimentant le cycle de la violence. Dans ce but, elles soutenaient les familles de personnes non inculpées mais frappées de restrictions s'appliquant à leur mobilité, à leurs contacts humains et à leurs moyens de communication pour un laps de temps non spécifié, et elles faisaient du lobbying pour libérer des personnes retenues par l'État britannique en détention préventive à long terme. Les femmes ont été impliquées dans l'évaluation de la politique Prevent et se sont élevées contre l'enregistrement d'informations personnelles et la vidéosurveillance de citoyens non suspects. Les activistes musulmanes ont donc apporté une contribution clé à la mise au point d'un ensemble de pratiques constituant une approche communautaire de l'antiterrorisme. En tant que réaction populaire de la base, ce travail a contribué de façon décisive à promouvoir un plus grand sentiment de sécurité, une perspective plus inclusive orientée sur la communauté, et une alternative aux pratiques sécuritaires pragmatiques mais souvent brutales de l'État.

\section{Des négociations sur le fil du rasoir}

Les femmes ont jugé bénéfiques cette mise en lumière de l'activisme et l'opportunité de s'engager de façon décisive avec l'État, de contribuer au développement des communautés locales, d'aider à prévenir la violence et à promouvoir la sécurité. Selon elles, de nombreuses raisons les poussent à s'engager : un sens du devoir islamique et civique, un désir de participer à la justice sociale et à la protection de leurs concitoyens, la volonté de saisir l'occasion de modeler et d'influencer les politiques sociales et politiques. Pourtant, cette politique a créé de nouveaux défis à relever. Au niveau macro, le discours négatif sur le danger inhérent aux communautés musulmanes et sur le besoin de les réguler a été renforcé et, avec lui, le concept d'un activisme social efficacement sécurisé. Certaines activistes craignent également qu'en travaillant sur des questions liées à l'antiterrorisme, elles ne confirment tacitement la construction problématique des femmes musulmanes - et des musulmans en général - au sein de la politique Prevent. L'État met en place son cadre sécuritaire du haut vers le bas, ce qui, en conjonction avec la création de groupes internes et externes, a créé des divisions au sein des communautés et entre les organisations de la base : devenues concurrentes dans la course aux financements, elles sont aussi encouragées à se vendre en tant qu'expertes et "modérées" par rapport aux autres organisations. Cette atmosphère a 
créé un sentiment de défiance au sein des communautés, avec des activistes négociant sur le fil du rasoir : conserver la crédibilité et la confiance de la base tout en travaillant avec des acteurs étatiques comme la police ou les conseils municipaux chargés d'appliquer la politique Prevent au niveau local. En outre, la suspicion s'est étendue vers l'intérieur - les femmes musulmanes activistes ayant elles-mêmes été ciblées par les agences de sécurité, ouvertement ou subrepticement. Les femmes engagées dans certains programmes visant à contrer l'extrémisme violent sont devenues des dangers potentiels. Les groupes intervenant auprès de femmes et de familles affectées par des activités extrémistes violentes ont été les premiers livrés à la suspicion de l'État. Une suspicion qui se traduit le plus
Dans le cas d'une organisation s'occupant de violence domestique, par exemple, l'intérêt tenait au présupposé plutôt étrange que ses usagers bénéficiaires - dont certaines étaient des femmes musulmanes - pouvaient être mariées à des extrémistes violents. souvent par l'exclusion de groupes aux points de vue politiques ou théologiques jugés indésirables : des femmes identifiées ou s'identifiant elles-mêmes comme salafistes ou islamistes ont été particulièrement stigmatisées dans les interprétations sécuritaires et de plus en plus rejetées par le gouvernement qui a sapé leur travail. La perception ambivalente des activistes femmes se perçoit encore dans cet exemple alarmant : des organisations de femmes impliquées dans le travail communautaire non lié à l'antiterrorisme ont été sollicitées pour participer au recrutement d'informateurs. Dans le cas d'une organisation s'occupant de violence domestique, par exemple, l'intérêt tenait au présupposé plutôt étrange que ses usagers bénéficiaires - dont certaines étaient des femmes musulmanes - pouvaient être mariées à des extrémistes violents. Ces expériences très négatives ont façonné le degré d'engagement des femmes musulmanes activistes avec l'État. Beaucoup nous ont fait part de leurs tentatives de réconcilier les objectifs du projet et le désir de travailler pour la sécurité publique, avec la peur et la stigmatisation associées à cette action. Souvent, ces pressions ont rendu leur travail intenable.

\section{Subvertir les discours sécuritaires}

Ces défis ont créé un contexte complexe dans lequel l'activisme des femmes musulmanes peut être à tout moment encouragé, jugé positif pour la sécurité de l'État ou bien rejeté. Leur récente exclusion de la nouvelle politique Prevent ${ }^{(13)}$ et le retrait du financement lié à la sécurité pour de nombreux projets communautaires créent 
une nouvelle série de problèmes. Les communautés musulmanes restent stigmatisées dans le discours sur la sécurité, alors même que les contributions des femmes musulmanes sont désormais liées à la cohésion sociale et non à la politique de sécurité. En outre, les activistes affiliées à des groupes étiquetés ou s'identifiant comme salafistes ou islamistes sont désormais totalement exclues des partenariats entre l'État et les communautés musulmanes, ce qui limite d'éventuelles contributions des approches communautaires à l'antiterrorisme.

Pourtant, malgré ce contexte défavorable, les activistes participant à la recherche conservent un optimisme prudent. La décennie qui a suivi le 11 septembre a apporté des opportunités tout en créant un traumatisme. Des leçons ont été apprises et une nouvelle assurance pour s'engager avec l'État en des termes égaux ont été cultivés. Les militantes musulmanes ont acquis de la visibilité sur des questions dépassant leur communauté religieuse ou ethnique. On est désormais conscient que l'agenda de l'État, dicté par la sécurité, exige l'engagement actif et informé d'un public plus large, notamment les citoyens les plus négativement affectés. Les femmes musulmanes occupent une position clé en tant que "minorité visible", mais aussi en tant qu'activistes sociales et politiques dans les partenariats entre les communautés musulmanes, la société britannique et l'État. Cette période de troubles a conduit à mettre en lumière l'existence d'une double appréhension de la sécurité, par l'État et par la communauté. La conviction que ces deux formes sont non seulement compatibles mais peuvent fonctionner en symbiose paraît bien enracinée. Il est clair, désormais, que la sécurité physique, sociale et psychologique de tous les citoyens est indispensable pour assurer la sécurité de l'État, et que l'injustice de l'État continuera à susciter de la colère et de la marginalisation. En contribuant à ces processus, les femmes musulmanes résistent aux préjugés de l'État britannique et de leurs propres communautés, pour défier la politique sécuritaire et promouvoir la sécurité pour tous.

De plus, l'expérience de ces activistes musulmanes soulève des questions plus vastes sur le mode d'engagement des individus avec ces structures qui les stigmatisent et les stéréotypent ostensiblement. Dans le cas des femmes musulmanes, une longue histoire d'imaginaire orientaliste, où le préjugé croise le genre, la religion, l'ethnicité et la politique, a informé et modelé la perception de l'État britannique et son interaction avec elles. Elles ont été dépeintes comme des victimes, comme des passerelles en direction des hommes, comme étant capables d'user d'un pouvoir familial dans une "guerre à la terreur" ouvertement masculine. Pourtant, de nombreuses activistes ont choisi de dialoguer avec ces représentations, qu'elles ont ainsi subvertis. Comme la notion d'imitation chez Bhabha ${ }^{(14)}$, l'acte même de l'Autre travaillant avec le pouvoir dominant perturbe et déstabilise la cohérence de discours sexistes ou racistes. L'image d'une femme musulmane passive, docile et victimisée vole en éclats quand celle-ci se 
lève en tant que citoyenne exigeant l'égalité, la justice et le changement. En prenant des risques calculés dans leur engagement avec ou contre l'État pour combattre la violence et la terreur par le biais d'agences étatiques et non étatiques, les femmes musulmanes non seulement façonnent les options sociales et politiques de la GrandeBretagne contemporaine, mais elles utilisent aussi l'essentialisation de leur moi suivant les catégories "femmes musulmanes" et "leaders de communautés" - à leur profit et au bénéfice de la société en général. Ce sont à la fois des actes de résistance et des actes de responsabilité sociale.

\section{Traduit de l'anglais par Françoise Bouillot}

\section{Notes}

1. Basia Spalek, Salwa El Awa et Laura Zara McDonald, et Robert Lambert, Police-Muslim Engagement and Partnerships for the Purposes of Counter-Terrorism: an Examination (Summary Report and Report), University of Birmingham with AHRC, 2008, 2009 ; Basia Spalek, Laura Zara McDonald et Salwa El Awa, Preventing Religio-Political Extremism Amongst Muslim Youth: a Study Exploring Police-Community Partnership, University of Birmingham with AHRC, 2011 ; Basia Spalek et Laura Zara McDonald, Connected Communities: Conflict with and between Communities: Exploring the Role of Communities in Helping to Defeat and/or Endorse Terrorism and the Interface with Policing Efforts to Counter Terrorism, University of Birmingham with AHRC, 2011.

2. Sara Danius, Stefan Jonsson et Gayatri Chakravorty Spivak, "An interview with Gayatri Chakravorty Spivak", in Boundary 2, vol. 20, $\mathrm{n}^{\circ}$ 2, 1993, p. 35 .

3. Equal Opportunities Commission (EOC), Moving on Up? Bangladeshi, Pakistani and Black Caribbean Women and Work, London, EOC, 2006 ; Lisa Buckner, Sue Yeandle and Sue Botcherby, Moving on up? Ethnic Minority Women and Work: Local Labour Markets, London, EOC \& University of Leeds, 2007.

4. Robert Lambert and Jonathan Githens-Mazer, Islamophobia and Anti-Muslim Hate Crime: UK Case Studies, University of Exeter, 2010.

5. Basia Spalek, Salwa El Awa, Laura Zara McDonald and Robert Lambert, op. cit.; Home Office, HM Government, CONTEST British Counter Terrorism Strategy, 2009, 2011 ; Home Office, HM Government, Prevent Strategy, 2008, 2011.

6. Basia Spalek, Laura Zara McDonald and Salwa El Awa, op. cit.

7. Home Office Statistical Bulletin, "Operation of Police Powers Under the Terrorism Act 2000 and Subsequent Legislation: Arrests, Outcomes and Stops \& Searches Great Britain 2009/10", 2010 ; Alpa Parmar, "Stop and Search in London: Counter-Terrorist or Counterproductive?", in Policing and Society, vol. 21, n 4, 2011, pp. 369-382.

8. David Cameron, Munich Security Conference, 2007, www.number10.gov.uk/news/speeches-andtranscripts/2011/02/pms-speech-at-munich-security-conference-60293.

9. Claudia Brunner, "Occidentalism Meets the Female Suicide Bomber: a Critical Reflection on Recent Terrorism Debates; a Review Essay", in Signs, vol. 32, n 4, 2007, pp.357-970.

10. Home Office, HM Government, Prevent Strategy, op. cit.

11. Home Office, HM Government, CONTEST British Counter Terrorism Strategy, op. cit., pp. 29 et 37.

12. Abdul Haqq Baker, Extremists in Our Midst: Confronting Terror, Basingstoke, Palgrave MacMillan, 2011.

13. Home Office, HM Government, CONTEST British Counter Terrorism Strategy, op. cit.

14. Homi K. Bhabha, "Of Mimicry and Man: The Ambivalence of Colonial Discourse", in The Location of Culture, London and New York, Routledge, 1994. Traduction française : "Du mimétisme et de l'homme : l'ambivalence du discours colonial", in Les Lieux de la culture, Paris, Payot, 2007. 